\title{
Spinal Processing of Noxious and Innocuous Cold Information: Differential Modulation by the Periaqueductal Gray
}

\author{
J. Lianne Leith, Stella Koutsikou, Bridget M. Lumb, and Richard Apps \\ Department of Physiology and Pharmacology, University of Bristol, Bristol BS8 1TD, United Kingdom
}

In addition to cold being an important behavioral drive, altered cold sensation frequently accompanies pathological pain states. However, in contrast to peripheral mechanisms, central processing of cold sensory input has received relatively little attention. The present study characterized spinal responses to noxious and innocuous intensities of cold stimulation in vivo and established the extent to which they are modulated by descending control originating from the periaqueductal gray $(\mathrm{PAG})$, a major determinant of acute and chronic pain. In lightly anesthetized rats, hindpaw cooling with ethyl chloride, but not acetone, was sufficiently noxious to evoke withdrawal reflexes, which were powerfully inhibited by ventrolateral (VL)-PAG stimulation. In a second series of experiments, subsets of spinal dorsal horn neurons were found to respond to innocuous and/or noxious cold. Descending control from the VL-PAG distinguished between activity in nociceptive versus non-nociceptive spinal circuits in that innocuous cold information transmitted by non-nociceptive class 1 and wide-dynamic-range class 2 neurons remained unaltered. In contrast, noxious cold information transmitted by class 2 neurons and all cold-evoked activity in nociceptive-specific class 3 neurons was significantly depressed. We therefore demonstrate that spinal responses to cold can be powerfully modulated by descending control systems originating in the PAG, and that this control selectively modulates transmission of noxious versus innocuous information. This has important implications for central processing of cold somatosensation and, given that chronic pain states are dependent on dynamic alterations in descending control, will help elucidate mechanisms underlying aberrant cold sensations that accompany pathological pain states.

\section{Introduction}

Understanding the neural mechanisms underlying cold sensation is important behaviorally, given the vital role of temperature perception in survival, but also clinically, given the aberrant cold responses frequently observed in neuropathic pain (Ochoa and Yarnitsky, 1994; Jorum et al., 2003). Peripheral mechanisms of cold somatosensation in relation to both acute and chronic pain states have received much recent interest. However, there is a surprising lack of knowledge regarding central processing of cold information, in particular whether cold-evoked responses at the spinal level are modulated by descending control systems, which are known to have powerful modulatory effects on other sensory modalities (Lovick and Bandler, 2005; Heinricher et al., 2009).

Innocuous cooling activates subsets of $\mathrm{A} \delta$ - and C-fiber lowthreshold afferents (Bessou and Perl, 1969; Leem et al., 1993; Campero et al., 1996). More intense cold stimuli additionally activate populations of nociceptive afferents (both $\mathrm{A} \delta$ - and C-fiber units), which display a range of activation thresholds and

\section{Received Jan. 8, 2010; revised Feb. 2, 2010; accepted Feb. 17, 2010.}

We gratefully acknowledge the financial support of the Biotechnology and Biological Sciences Research Council, the technical assistance of Barbara Carruthers and Rachel Bissett, and Dr. Lucy Donaldson and Dr. James Dunham for comments on the manuscript.

Correspondence should be addressed to Dr. Bridget M. Lumb, Department of Physiology and Pharmacology, University of Bristol, School of Medical Sciences, University Walk, Bristol BS8 1TD, UK. E-mail: B.M.Lumb@ bristol.ac.uk.

DOI:10.1523/JNEUROSCI.0122-10.2010

Copyright $\odot 2010$ the authors $\quad 0270-6474 / 10 / 304933-10 \$ 15.00 / 0$ encode changes in stimulus intensity (LaMotte and Thalhammer, 1982; Lynn and Carpenter, 1982; Leem et al., 1993; Simone and Kajander, 1996, 1997; Cain et al., 2001). At the spinal level, electrophysiological studies have demonstrated that wide-dynamicrange and nociceptive-specific neurons in the dorsal horn are excited by cold stimuli and encode intensity to noxious cold over a wide range of temperatures (Christensen and Perl, 1970; Khasabov et al., 2001; Brignell et al., 2008). Additionally, cold stimulation of the hindpaw or face evokes intensity-dependent Fos expression in spinal and medullary dorsal horn neurons, respectively (Strassman et al., 1993; Abbadie et al., 1994; Doyle and Hunt, 1999; Todd et al., 2005).

Spinal processing of sensory information is subject to dynamic descending modulation from supraspinal structures, which is a major determinant of the acute pain experience evoked by noxious mechanical and heat stimuli (Millan, 2002; Heinricher et al., 2009). In particular, the periaqueductal gray (PAG) is a key source of descending control that operates in different behavioral states (Keay and Bandler, 2001; Lovick and Bandler, 2005), and it is now recognized that chronic pain states are dependent on descending control from brainstem centers, including the PAG (Pertovaara et al., 1996; Urban and Gebhart, 1999; Pertovaara, 2000; Monhemius et al., 2001; Pertovaara and Wei, 2003; Vanegas and Schaible, 2004; Carlson et al., 2007). Descending control from the PAG profoundly modulates processing of mechanical and heat information (Heinricher et al., 2009). However, it re- 
mains unknown to what extent processing of cold information is subject to descending control.

By recording noxious cold-evoked withdrawal reflexes and dorsal horn neuronal activity in response to both innocuous and noxious cold stimuli, the present study provides direct evidence that descending control from the ventrolateral (VL)-PAG selectively modulates transmission of noxious cold information. Dynamic alterations in descending control underlie central sensitization and chronic pain states; therefore, changes in descending modulation of cold inputs during the transition from acute to chronic pain may contribute to aberrant responses to cold that accompany neuropathic pain.

\section{Materials and Methods}

\section{Animal preparation}

All experiments were performed in accordance with the UK Animals (Scientific Procedures) Act 1986 and associated guidelines. Male adult Wistar rats (280-320 g; $n=38$; Harlan) were housed in standard conditions and handled frequently to minimize stress on the day of the experiment.

Anesthesia was induced using $4 \%$ halothane in $\mathrm{O}_{2}$, and the jugular vein was cannulated for anesthetic maintenance using a constant intravenous infusion of alphaxalone $\left(\sim 25 \mathrm{mg} \cdot \mathrm{kg}^{-1} \cdot \mathrm{h}^{-1}\right.$ Alfaxan; Jurox). The carotid artery was exposed and cannulated for recording of blood pressure, and the trachea was intubated. Body temperature was maintained within physiological limits by means of a feedback-controlled heating blanket and rectal probe. In animals in which dorsal horn neuronal activity was to be recorded, a laminectomy was performed between T11 and T13 to expose the lumbar spinal cord. Animals were then positioned in a stereotaxic frame and a craniotomy was performed to allow access to the PAG with glass micropipettes.

In neuronal recording experiments, anesthesia was maintained at a level at which there were no precipitous changes in blood pressure in response to minor noxious stimuli, and in electromyographic (EMG) recording experiments it was reduced to a level at which animals were moderately responsive to firm pinch of the contralateral forepaw. Animals were allowed to stabilize at these levels for a minimum of $30 \mathrm{~min}$ before recording of neuronal or EMG activity.

\section{Recording of skin temperature}

Surface skin temperature on the hindpaw was recorded using a K-type thermocouple (Physitemp) held in place with a small dab of cyanoacrylate glue (distant from the site of stimulation; Loctite; Henkel). The thermocouple was connected to a digital thermometer (BAT-12; Physitemp), and the output signal was digitized using a 1401plus data acquisition system (Cambridge Electronic Design). Subcutaneous skin temperature was recorded using a T-type thermocouple (made in-house) connected to a digital thermometer, and then the output signal was digitized via a 1401 plus.

\section{Recording of EMG activity}

An intramuscular bipolar electrode, custom made from two short lengths of Teflon-coated, 0.075-mm-diameter, stainless steel wire (Advent Research Materials), was inserted into the biceps femoris of the left hind leg to record EMG activity during the withdrawal reflex. The EMG signal was amplified $(\times 5000)$ and filtered $(50 \mathrm{~Hz}$ to $5 \mathrm{kHz}$; NeuroLog System; Digitimer), before being captured for subsequent analysis via a 1401plus (Cambridge Electronic Design) onto a PC running Spike2 version 5 software (Cambridge Electronic Design). The magnitude of the withdrawal reflex evoked by thermal stimuli was quantified by measuring the modulus of the EMG using Spike2 software. This value was then corrected for background noise by subtracting noise over the same length of time as the response (measured before application of the thermal stimulus). In some experiments, surface and subsurface skin temperatures were recorded simultaneously, and it was therefore possible to measure the threshold temperature at which the withdrawal reflex occurred in addition to response magnitude. If no EMG was observed following
PAG stimulation, withdrawal threshold was assigned as $0^{\circ} \mathrm{C}$ for data analysis.

\section{Recording of dorsal horn neuronal activity}

The vertebral column was clamped at each end of the laminectomy to maximize stability during neuronal recordings. The dura was removed, a pool was made with skin flaps, and the whole area was filled with agar to further stabilize the preparation. Once set, a small window was cut out of the agar over the desired recording site and filled with warm paraffin oil. A glass-coated tungsten microelectrode $(\sim 5 \mathrm{M} \Omega$; Melanie Ainsworth; Northamptonshire, UK) was lowered into the cord. Extracellular singleunit neuronal activity was amplified $(\times 5000)$ and filtered $(500 \mathrm{~Hz}$ to 10 $\mathrm{kHz}$; NeuroLog System; Digitimer) before being captured at $10 \mathrm{kHz}$ for subsequent analysis via a 1401plus (Cambridge Electronic Design) onto a PC running Spike2 version5 software (Cambridge Electronic Design).

\section{Functional classification of spinal neurons}

Single units were isolated using gentle tapping, stroking, or firm pinch of the hindlimb, delivered manually, as a search stimulus. It was not feasible to use cooling as a search stimulus; therefore, it must be borne in mind that the search stimuli used biased the population to mechanically sensitive neurons.

Once a unit had been identified, the peripheral receptive field was characterized using low-threshold (brush, tap) and high-threshold (pinch) mechanical stimuli. According to their mechanical response properties, units were classified as class 1 (low threshold, non-noxious), class 2 (low and high threshold, wide dynamic range), or class 3 (high threshold, nociceptive specific) (Menetrey et al., 1977; 1979). Neurons were then tested for responsiveness to the following thermal stimuli: noxious heat $\left(50^{\circ} \mathrm{C}\right.$ water; $\left.1 \mathrm{ml}\right)$, innocuous cooling (acetone; $\left.1 \mathrm{ml}\right)$, or noxious cold [ethyl chloride (EC); $1 \mathrm{ml}$ ].

Responses to acetone and EC were quantified by counting the total number of spikes evoked until activity returned to the prestimulus level; this value was then corrected for spontaneous activity of the neuron over the same length of time as the response (measured before application of the thermal stimulus). In some neurons, afferent input was further characterized by monitoring responses to percutaneous electrical stimuli ( 1 ms square pulse) delivered to the center of the receptive field via needle electrodes. Thresholds for A- and C-fiber activation were established and repeated sweeps were made at both 1.5 and 3 times $\mathrm{C}$-fiber threshold voltage (a train of $201 \mathrm{~ms}$ square pulses delivered at $0.1 \mathrm{~Hz}$ ). All neurons tested showed responses at latencies consistent with input from both Aand $\mathrm{C}$-fiber afferents and were therefore classified as $\mathrm{C}$ positive (Waters and Lumb, 2008).

\section{Antidromic testing of spinal neurons for a supraspinal projection} In some experiments, dorsal horn neurons were tested for a supraspinal projection to the caudal brainstem. Supraspinal projection neurons were identified by their antidromic responses to electrical stimulation in the vicinity of the contralateral inferior olivary complex $[\sim 12.5 \mathrm{~mm}$ caudal to bregma, $1.2-1.5 \mathrm{~mm}$ lateral to the midline, and $8.5-9.0 \mathrm{~mm}$ deep to the cortical surface according to the brain atlas of Paxinos and Watson (2005)] using a bipolar stimulating electrode (interpolar distance of 0.5 mm; SNE-100X; Harvard Apparatus). Single pulses (20-100 $\mu$ A, $0.1 \mathrm{~ms}$ duration, at a rate of $0.1 \mathrm{~Hz}$ ) were delivered via the stimulating electrode, and dorsal horn neurons were classified as projection neurons if they met the following standard criteria for antidromic activation: (1) an all-ornone response to stimulation, (2) constant latency responses, (3) frequency following to three stimuli delivered at a rate of $100 \mathrm{~Hz}$, and (4) collision of the antidromic spike with a spontaneous or evoked orthodromic spike (Lipski, 1981).

\section{Cold stimulation of the skin}

Two different cooling stimuli were used. One milliliter of either 100\% EC (Acorus Therapeutics) or 100\% acetone (Fisher Scientific) was applied topically to the hindpaw (in withdrawal reflex experiments) or the hindlimb receptive field (in dorsal horn neuronal recording experiments) using a pipette. Care was taken not to touch the skin with the pipette tip. Because of the position of the animal in the stereotaxic frame, the pipette 
was aimed at the lateral paw, but the coolant very rapidly $(<1 \mathrm{~s})$ spread over the whole paw, including both dorsal and plantar surfaces.

\section{Neuronal activation in the PAG}

Glass micropipettes (Harvard Apparatus) were driven stereotaxically into the VL-PAG at $\sim 7.6-7.8 \mathrm{~mm}$ caudal to bregma, $0.8-1.0 \mathrm{~mm}$ lateral to midline, and $5.3-5.5 \mathrm{~mm}$ deep to the cortical surface according to the brain atlas of Paxinos and Watson (2005). Micropipettes were filled with a $50 \mathrm{~mm}$ solution of the excitatory amino acid DL-homocysteic acid (DLH; Sigma) containing pontamine sky blue dye (Gurr) to mark injection sites. Microinjections of DLH (60-80 nl) were given under microscopic guidance using a custom-made, paraffin-filled pressure injection system attached to a $1 \mu \mathrm{l}$ syringe (SGE Analytical Science). The concentration of DLH was the same as that used in previous studies of descending control from the PAG (Waters and Lumb, 1997; McMullan and Lumb, 2006a, 2006b; Koutsikou et al., 2007; Waters and Lumb, 2008), and since only very small amounts of DLH were injected, it is likely that its administration results in excitation of PAG neuronal cell bodies rather than depolarizing block (Lipski et al., 1988). Consistent with previous studies (see above), microinjections into the VL-PAG evoked transient decreases in mean arterial pressure (data not shown).

\section{Experimental protocol}

Descending modulation of cold-evoked withdrawal reflexes. Following preparatory surgery, a glass micropipette containing DLH solution was lowered vertically into the left VL-PAG (ipsilateral to the stimulated hindpaw). Flexion withdrawal reflexes were recorded in response to EC delivered to the left hindpaw at $5 \mathrm{~min}$ intervals. After three "baseline" withdrawal responses to EC were recorded, an injection of DLH was made into the VL-PAG. EC was applied again to the paw $10 \mathrm{~s}$ after DLH injection ("PAG" in bar charts) and responses to three further applications were conducted at $5 \mathrm{~min}$ intervals (at 5,10 , and $15 \mathrm{~min}$ post-DLH microinjection, termed "PAG +5 min," "PAG + 10 min," and "PAG + 15 min," respectively, in bar charts).

Descending modulation of cold-evoked neuronal activity. Following isolation of a neuron and characterization of its receptive field and response properties, a glass micropipette containing DLH solution was lowered vertically into the left VL-PAG (ipsilateral to the stimulated hindlimb). Neuronal activity was recorded in response to acetone or EC delivered to the receptive field of the neuron at $5 \mathrm{~min}$ intervals. After three baseline neuronal responses to acetone or EC were recorded, an injection of DLH was made into the VL-PAG. Acetone or EC was applied again to the receptive field $10 \mathrm{~s}$ after DLH injection (PAG in bar charts), and responses to three further applications were conducted at $5 \mathrm{~min}$ intervals (at 5, 10, and 15 min post-DLH microinjection; termed PAG +5 min, PAG $+10 \mathrm{~min}$, and PAG $+15 \mathrm{~min}$, respectively, in bar charts).

\section{Histology}

At the end of experiments, animals were killed by overdose of sodium pentobarbital (30 mg i.v. bolus; Sigma). The brain was removed, fixed for $24 \mathrm{~h}$ in paraformaldehyde solution ( $4 \%$ in $0.1 \mathrm{M}$ phosphate buffer), and then cryoprotected in 30\% sucrose solution for $24 \mathrm{~h}$. Brains were sectioned at $60 \mu \mathrm{m}$ and PAG injection sites marked with pontamine sky blue dye were localized with reference to the rat brain atlas of Paxinos and Watson (2005) and plotted onto standard transverse diagrams of the midbrain.

\section{Data analysis}

All EMG and neuronal activity data are displayed as mean \pm SEM. All statistical analysis was performed using Prism 4 (GraphPad). Post-DLH (PAG on bar charts) and recovery responses $(\mathrm{PAG}+5 \mathrm{~min}, \mathrm{PAG}+10$ min, and PAG +15 min on bar charts) to acetone or EC were compared with baseline responses using Kruskal-Wallis test followed by Dunn's multiple comparison test. Significance was taken at the $5 \%$ level.

\section{Results}

\section{Acetone and ethyl chloride evoke distinct skin} cooling profiles

In the present study, two different chemical coolants, acetone and EC, were used to produce different intensities of cooling. These

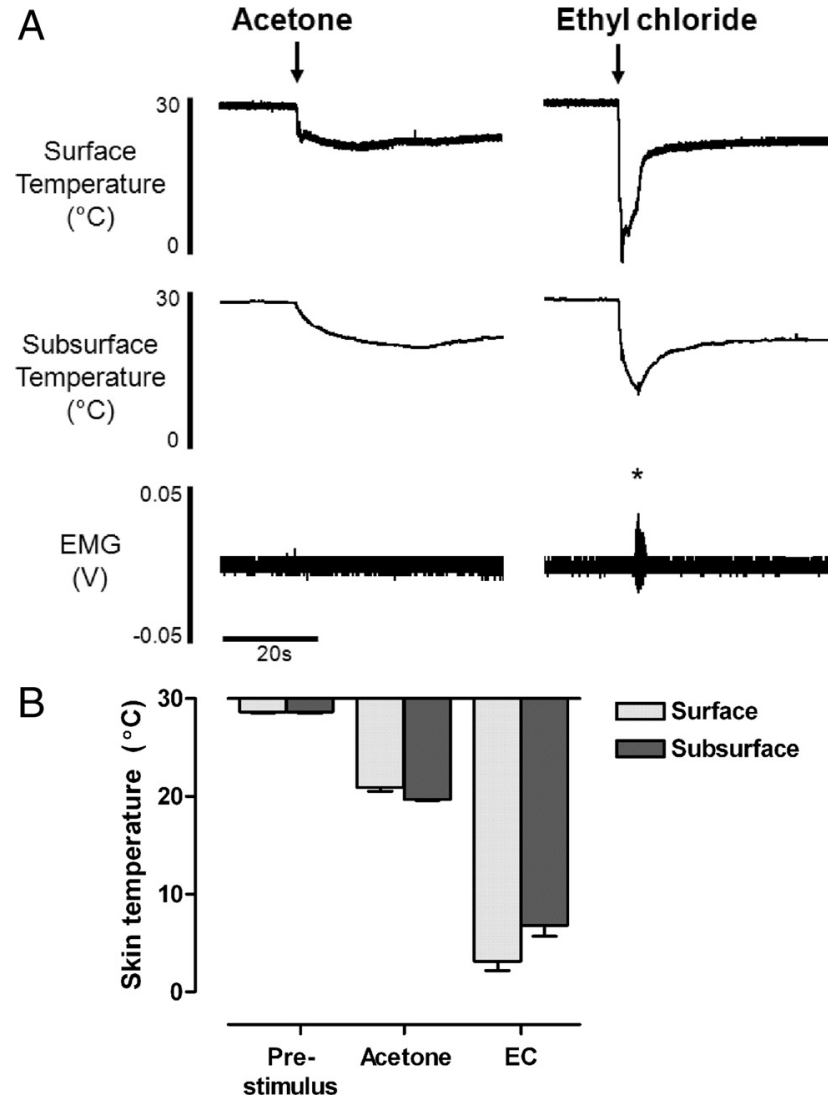

Figure 1. Acetone and ethyl chloride evoke very different skin cooling profiles in vivo. $\boldsymbol{A}$, Example traces of surface and subsurface skin temperature measurements following application of acetone and EC to the hindpaw. Note that ethyl chloride evokes withdrawal reflexes in lightly anesthetized animals [measured as EMG activity from biceps femoris; marked with asterisk $\left({ }^{*}\right)$ ], whereas acetone does not. $\boldsymbol{B}$, Bar chart showing prestimulus skin temperatures and maximum surface and subsurface temperature changes following hindpaw acetone and EC application (mean \pm SEM; 7 trials from $n=3$ animals).

stimuli have been used in behavioral studies of cold somatosensation; however, the skin temperature changes evoked are rarely measured (cf. Colburn et al., 2007). We therefore recorded surface and subsurface skin temperature changes during topical application of both acetone and EC to the hindpaw in vivo (Fig. 1). Both stimuli generated rapid changes in surface and subsurface skin temperature; however, the magnitude of temperature change was significantly different. Acetone lowered surface and subsurface skin temperature by 7.7 and $8.9^{\circ} \mathrm{C}$, respectively (surface $28.6 \pm 0.1$ to $20.9 \pm 0.4^{\circ} \mathrm{C}$, and subsurface $28.6 \pm 0.1$ to $19.7 \pm 0.1^{\circ} \mathrm{C} ; 7$ trials from $n=3$ animals) (Fig. $1 B$ ). In contrast, EC application evoked significantly colder skin temperatures than acetone $(p<0.001$, Kruskal-Wallis test and Dunn's multiple comparison test) (Fig. $1 B$ ), lowering surface and subsurface skin temperature by 25.4 and $21.8^{\circ} \mathrm{C}$, respectively (surface $28.6 \pm 0.1$ to $3.2 \pm 1.0^{\circ} \mathrm{C}$, and subsurface $28.6 \pm 0.1$ to $6.8 \pm 1.1^{\circ} \mathrm{C} ; 7$ trials from $n=3$ animals) (Fig. $1 B$ ). Skin temperature returned to prestimulus levels within the interstimulus interval of $5 \mathrm{~min}$ following both types of stimulus.

Cold stimulation of the paw with EC consistently evoked robust withdrawal reflexes in lightly anesthetized animals (measured as hindlimb EMG activity) (Figs. $1 A, 2 A$ ), suggesting that EC produces noxious cooling of the skin. The mean threshold of EC-evoked withdrawal reflexes was $6.2 \pm 0.6^{\circ} \mathrm{C}$ at the skin surface and $16.1 \pm 0.7^{\circ} \mathrm{C}$ subcutaneously ( 9 trials from $n=3$ animals) (Fig. 2C). In addition, EC application frequently evoked 
A
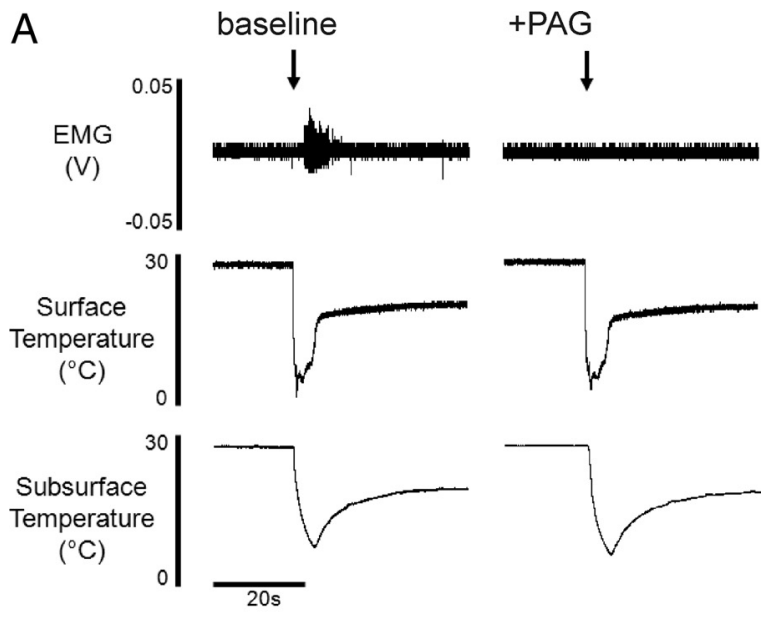

B

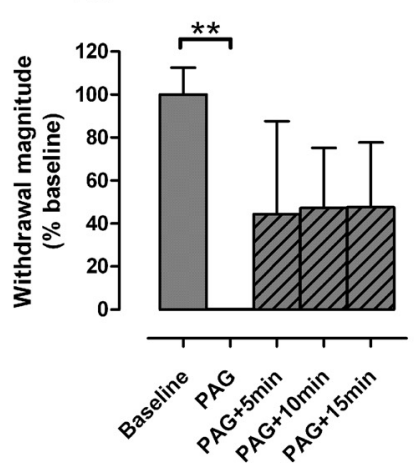

C

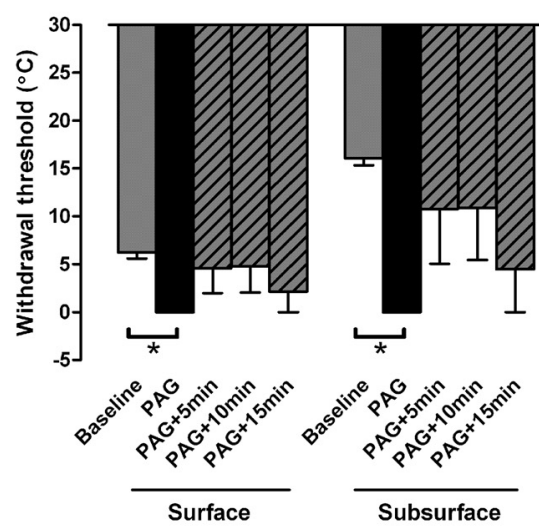

D

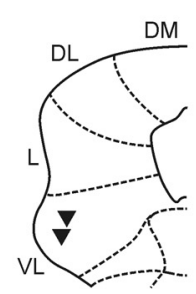

$-7.56$

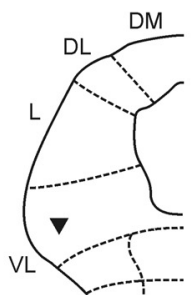

$-7.92$
$-8.28$

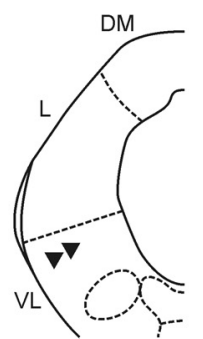

Figure 2. Noxious cold-evoked withdrawal reflexes are depressed by VL-PAG stimulation. A, Typical examples of EC-evoked withdrawal reflexes (measured as EMG activity) with corresponding surface and subsurface skin temperature measurements before and after stimulation of the VL-PAG; the withdrawal reflex is abolished by PAG stimulation, however the profile of skin cooling remains unaltered. $\boldsymbol{B}, \boldsymbol{C}$, The effects of VL-PAG stimulation on withdrawal reflex magnitude $(n=5)(\boldsymbol{B})$ and threshold (surface and subsurface; both $n=3$ ) (C). Data are expressed as mean \pm SEM. Statistical analysis compared post-DLH (PAG) and recovery (PAG $+5 \mathrm{~min}, \mathrm{PAG}+10 \mathrm{~min}, \mathrm{PAG}+15 \mathrm{~min}$ ) groups with baseline responses using Kruskal-Wallis test followed by Dunn's multiple comparison test; ${ }^{*} p<0.05 ;{ }^{* *} p<0.01$. D, Injection sites in the PAG from which the effects of PAG stimulation on EC-evoked responses were tested; coordinates are relative to bregma (Paxinos and Watson, 2005). DM, Dorsomedial; DL, dorsolateral; L, lateral; VL, ventrolateral. increases in mean arterial blood pressure, indicative of a noxious intensity of stimulation (data not shown). In the preparation used here, noxious intensities of stimulation are required to evoke withdrawal reflexes (McMullan et al., 2004), which indicates that EC is largely a noxious cold stimulus. In contrast, in the same animals withdrawal reflexes were never observed following acetone application, nor did acetone evoke any changes in blood pressure, suggesting it produces innocuous cooling of the skin. Nonetheless, it is likely that the temperature changes produced by acetone application will activate a small proportion of nociceptive afferents, since some cold-responsive nociceptors are reported to have activation thresholds of $>20^{\circ} \mathrm{C}$ (although most have thresholds at much colder temperatures) (Cain et al., 2001; Campero et al., 1996; Simone and Kajander, 1996). Clearly, however, activation of such afferents is not generally sufficient to produce overt behavioral responses to acetone, either in lightly anesthetized rats (this study) or in awake normal rats, which show little or no response to acetone (Choi et al., 1994; Decosterd and Woolf, 2000; Kim et al., 2009; Hulse et al., 2010); therefore, we consider acetone to be predominantly (though not exclusively) an innocuous cooling stimulus.

\section{Cold-evoked withdrawal reflexes are significantly depressed} by VL-PAG stimulation

Descending control originating in the PAG has been shown to powerfully modulate withdrawal reflexes evoked by noxious mechanical and thermal (heat) stimuli (e.g., Mayer et al., 1971; Fardin et al., 1984; Carstens et al., 1990; McMullan and Lumb, 2006a; Leith et al., 2007). However whether this control extends to noxious cold stimulation remains unclear; therefore, the effect of VL-PAG stimulation on cold-evoked withdrawal thresholds and response magnitudes was investigated.

PAG stimulation strongly depressed cold-evoked withdrawal reflexes in vivo to the extent that the responses were frequently abolished (Fig. 2). Cold-evoked withdrawal reflex magnitude was significantly reduced to $0.1 \pm 0.1 \%$ of baseline $(p<0.01, n=5$; Kruskal-Wallis test and Dunn's multiple comparison test) (Fig. $2 B$ ), and withdrawal threshold temperature was significantly colder (from $6.2 \pm 0.6^{\circ} \mathrm{C}$ at the skin surface and $16.1 \pm 0.7^{\circ} \mathrm{C}$ s.c. to $0^{\circ} \mathrm{C}$ at both the surface and the subsurface; both $p<0.05, n=$ 3; Kruskal-Wallis test and Dunn's multiple comparison test) (Fig. 2C). Reflex magnitude and threshold recovered partially during the 15 min period following PAG stimulation (Fig. 2 B, C).

Although stimulation of the PAG has been shown to alter cardiovascular parameters (Carrive, 1993; Bandler et al., 2000) that might alter the rate and extent of cooling produced by the stimulus, the profile of skin cooling evoked by EC was not altered following PAG stimulation (Fig. 2A). This suggests that the depression of withdrawal reflexes is caused mainly by direct central modulatory effects on neuronal excitability rather than being secondary to changes in peripheral blood flow.

The data therefore provide evidence that cold-evoked withdrawal reflexes can be strongly modulated by descending control from the VL-PAG. However, given that acetone does not evoke withdrawal reflexes in lightly anesthetized preparations, this approach does not allow us to test whether the PAG also modulates responses to innocuous cold. We therefore examined the activity of dorsal horn neurons in the spinal cord in response to both acetone and EC and whether this activity could be altered following stimulation of the PAG. 
A

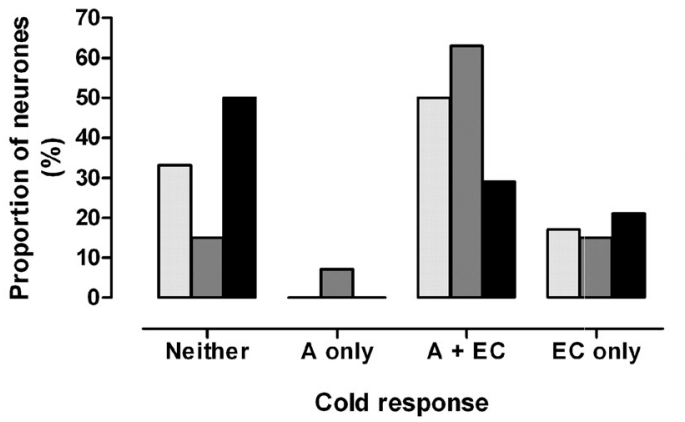

B

Acetone<smiles>I</smiles>

$\overline{8}$
on
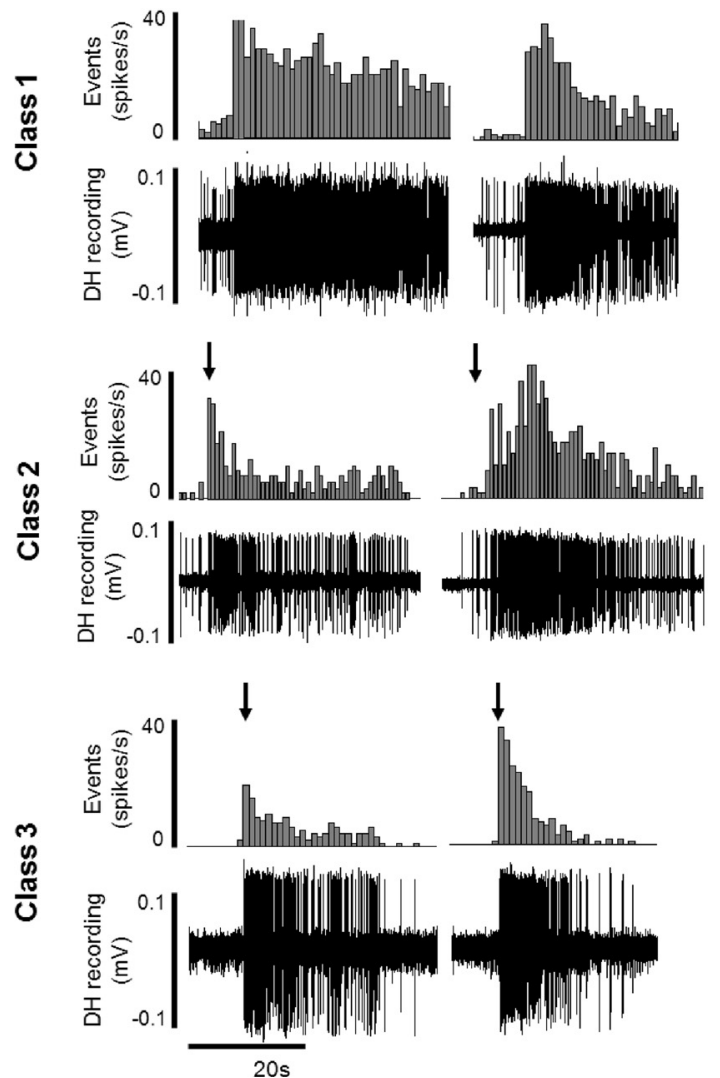
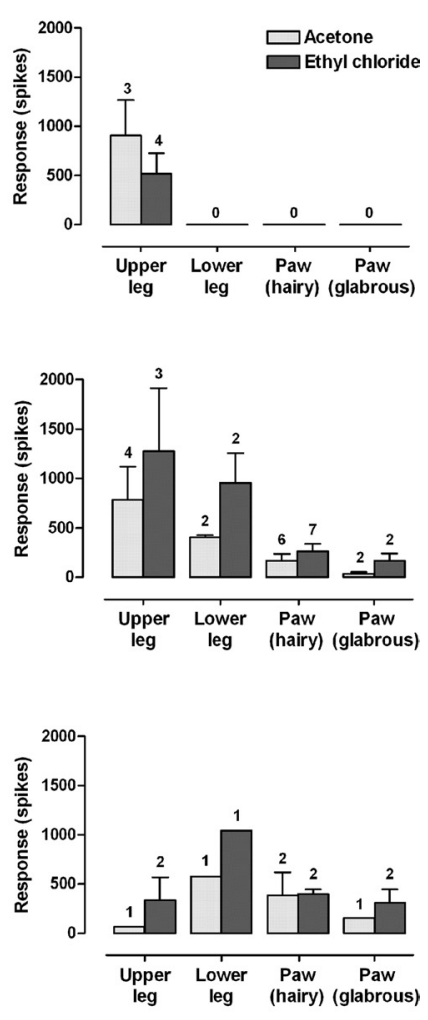

Figure 3. Subpopulations of class 1,2 , and 3 dorsal horn neurons respond to acetone and ethyl chloride. $A$, Summary of the proportions of class 1, 2, and 3 neurons that responded to acetone and/or EC. $\boldsymbol{B}$, Typical examples of responses to acetone and EC in cold-responsive class 1, 2, and 3 neurons (in each example the two sets of responses are recorded from the same neuron; raw dorsal horn (DH) recordings and corresponding peristimulus time histograms (spikes per $1 \mathrm{~s}$ bin) are shown). Note, however, that the majority of class 3 neurons did not respond to acetone. Arrows indicate onset of cold stimulus. $\boldsymbol{C}$, For each neuron class, responses (mean spikes \pm SEM) to the two cold stimuli are grouped in relation to receptive field location; numbers above each bar represent sample size.

\section{Subsets of class 1, 2, and 3 spinal neurons respond to acetone} and ethyl chloride

A total of 47 lumbar dorsal horn neurons (mean depth in cord $729 \pm 40 \mu \mathrm{m}$; range $280-1430 \mu \mathrm{m}$ ) were studied and classified by responses to low- and high-threshold mechanical stimulation of the receptive field according to the scheme defined by Menetrey et al. (1977); class 1, $n=6$; class 2, $n=27$; and class 3, $n=14$. Subsets of neurons in each class (class $1, n=2$; class 2, $n=$ 8 ; class $3, n=7$ ) were antidromically identified as supraspinally projecting, at least to the level of the caudal brainstem. There was no distinction between a neuron's response to cold stimuli and whether or not a supraspinal projection could be detected.
Response characteristics of all spinal neurons to acetone and EC are summarized in Figure 3. The response of each neuron to each type of cold stimulus was consistent from trial to trial. The majority $(66 \% ; 4 / 6)$ of non-nociceptive class 1 neurons responded to cold [acetone only, $n=$ $0(0 \%)$; acetone and EC, $n=3(50 \%)$; EC only, $n=1(17 \%)$; neither $n=2(33 \%)]$ (Fig. 3A). Class 1 neurons tended to show prolonged, high-frequency responses to both acetone and EC. In the three neurons that responded to both stimuli, responses to EC were always similar to or less than the responses to acetone (Fig. $3 B, C$, top panels) suggesting, as expected, that these neurons are not able to encode stimulus intensity into the noxious range. This indicates that the EC response in class 1 neurons represents the innocuous cooling component of the stimulus, since EC will activate low-threshold, cooling-sensitive afferents in addition to those in the nociceptive range.

The majority $(85 \% ; 23 / 27)$ of widedynamic-range class 2 neurons responded to cold [acetone only, $n=2(7 \%)$; acetone and EC, $n=17$ (63\%); EC only, $n=4$ (15\%); neither, $n=4$ (15\%)] (Fig. $3 A$ ). Class 2 neurons showed a range of response magnitudes to both acetone and EC that appeared to be related to receptive field location; neurons with receptive fields on proximal regions of the hindlimb (e.g., thigh) tended to show greater responses to cold than those with distal receptive fields (e.g., paw) (Fig. 3C, middle panel). This is unlikely to be caused by a different skin-cooling profile, since temperature measurements at the thigh were not significantly different compared with those at the paw (data not shown). Instead, the greater responses of neurons with proximal receptive fields are likely to be due to the higher degree of convergence of input in the spinal cord from peripheral afferents (Willis and Coggeshall, 1991). All class 2 neurons that were responsive to both cold stimuli showed greater responses to EC than acetone (Fig. $3 B, C$, middle panels), indicative of their ability to encode stimulus intensity into the noxious range (Khasabov et al., 2001).

Only half of class 3 neurons $(50 \% ; 7 / 14)$ responded to cold stimuli [acetone only, $n=0(0 \%)$; acetone and EC, $n=4(29 \%)$; EC only, $n=3$ (21\%); neither, $n=7$ (50\%)] (Fig. $3 A)$. In class 3 neurons, unlike class 2 , there was no clear relationship between receptive field location and response magnitude, and they did not encode stimulus intensity to the same extent, although it should be noted that the sample size of class 3 neurons is small (Fig. $3 C$, bottom panel). A large proportion of class 3 neurons did not respond to either acetone or EC. However, it remains possible that these neurons are cold responsive because the stimulus used 
in this study may not have achieved temperatures adequate for their activation. Somewhat unexpectedly, a small proportion $(4 / 14 ; 29 \%)$ of nociceptivespecific class 3 neurons (as classified by mechanical stimulation) also responded to acetone. However, as stated above, the skin temperatures produced by acetone application $\left(\sim 20^{\circ} \mathrm{C}\right)$ are likely to be sufficient to activate a small proportion of nociceptive afferents that could, in turn, activate nociceptive-specific class 3 neurons in the spinal cord. Therefore, acetone responses observed in class 3 neurons may indeed represent nociceptive activity (see Discussion).

An important additional consideration when interpreting these data is that both the cooling stimuli used here involve a low-threshold mechanical component (i.e., application of liquid to the skin). It is therefore possible that part of the response observed was due to stimulation of mechanically sensitive afferents. To determine the extent of firing evoked solely by this mechanical stimulus, a control stimulus of the same volume of room temperature water was applied to the receptive field of some neurons $(n=9$; data not shown). In class 1 and 2 neurons, room temperature water evoked a brief $(<1 \mathrm{~s})$, modest increase $(<10$ spikes) in firing, but no response was observed in class 3 neurons, which indicates that the response to acetone and EC (typically of long duration and high firing frequency) (Fig. 3) was predominantly due to skin cooling rather than any mechanical stimulation.

\section{Descending control from the PAG}

modulates spinal neuronal responses to noxious but not innocuous cold

Responses of non-nociceptive class 1 neurons to both acetone and EC remained unaltered after stimulation of the VLPAG, as shown in Figure 4 [117 \pm 63 and $122 \pm 16 \%$ of baseline responses; $n=3$ and $n=4$ (including one projection neuron), respectively; both $p>0.05$, KruskalWallis test and Dunn's multiple comparison test], indicating that descending control from the PAG does not significantly alter responses to innocuous cold in these neurons.

Similarly, stimulation of VL-PAG did not significantly alter responses of wide-dynamic-range class 2 neurons to acetone as shown in Figure 5 [123 $\pm 45 \%$ of baseline responses; $n=6$ (none positively identified as projection neurons); $p>0.05$, KruskalWallis test and Dunn's multiple comparison test]. However, responses of class 2 neurons to EC were significantly reduced by VL-PAG stimulation [to $17 \pm 7 \%$ of baseline; $n=7$ (including two projection neurons); $p<0.01$, Kruskal-Wallis test and Dunn's multiple comparison test] (Fig. 5), indicating that descending control from the PAG selectively depresses responses to

B

C
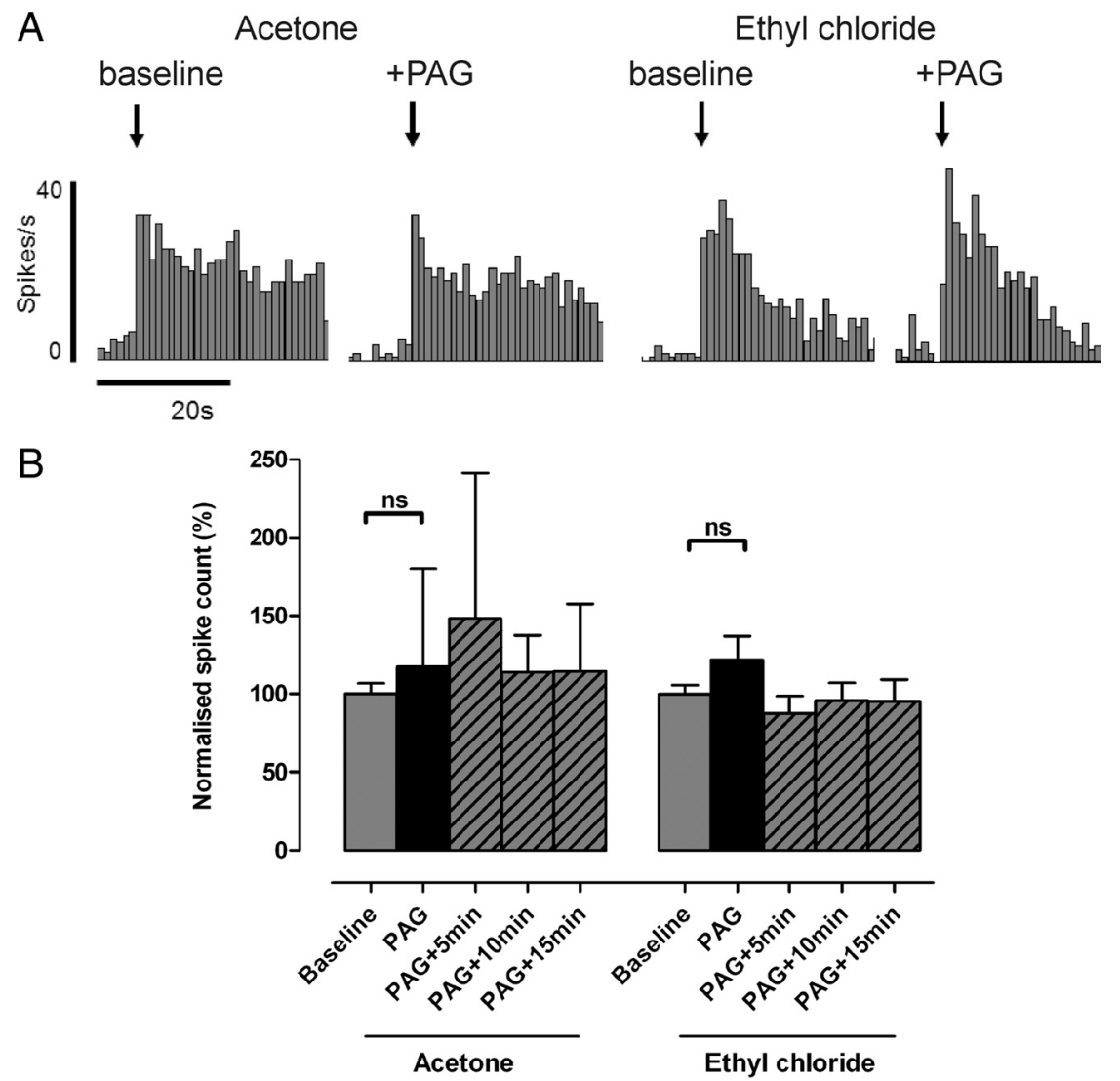

Figure 4. Cold-evoked activity remains unaltered in non-nociceptive class 1 neurons following PAG stimulation. $\boldsymbol{A}$, Typical examples of a class 1 neuron response to acetone and EC [recorded from the same neuron; peristimulus time histograms (spikes per $1 \mathrm{~s} \mathrm{bin)}$ are shown] before and after PAG stimulation. $\boldsymbol{B}$, The effect of VL-PAG stimulation on class 1 neuronal responses to acetone $(n=3)$ and EC $(n=4)$. Data are expressed as mean \pm SEM of normalized spike counts in response to acetone or EC. Statistical Kruskal-Wallis test followed by Dunn's multiple comparison test; $n s, p>0.05$. C, Injection sites in the PAG from which the effects of PAG stimulation on acetone- and/or EC-evoked responses were tested; coordinates are relative to bregma (Paxinos and Watson, 2005). DM, Dorsomedial; DL, dorsolateral; L, lateral; VL, ventrolateral.

noxious versus innocuous cold in these neurons. EC responses recovered to baseline levels within 5 min of VL-PAG stimulation $(\mathrm{PAG}+5 \mathrm{~min}, \mathrm{PAG}+10 \mathrm{~min}$, and PAG $+15 \mathrm{~min}$, all $p>0.05$ compared with baseline, Kruskal-Wallis test and Dunn's multiple comparison test) (Fig. 5).

Stimulation of VL-PAG strongly depressed cold-evoked activity in all nociceptive-specific class 3 neurons tested; responses to EC were significantly depressed as shown in Figure 6 [to $14 \pm 11 \%$ of baseline responses; $n=4$ (including two projection neurons); $p<0.01$, Kruskal-Wallis Test and Dunn's multiple comparison test]. These responses recovered only partially during the $15 \mathrm{~min}$ period following VL-PAG stimulation and remained significantly lower than baseline levels 15 min after VL-PAG stimulation 
A
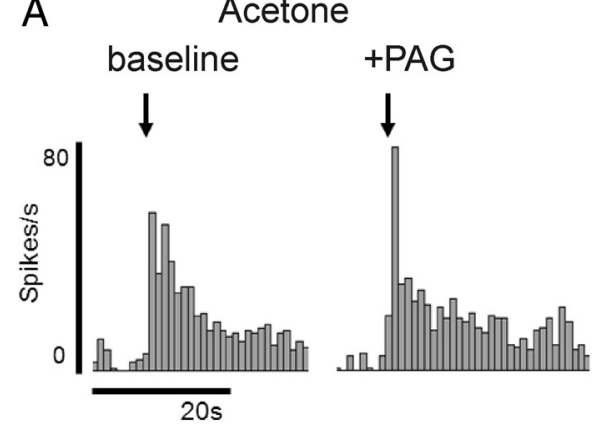

B

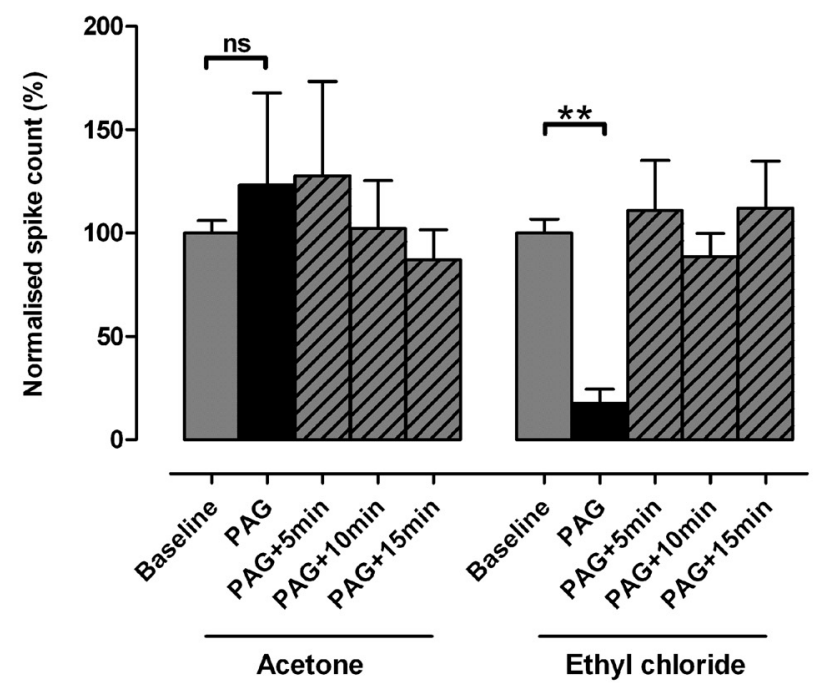

C
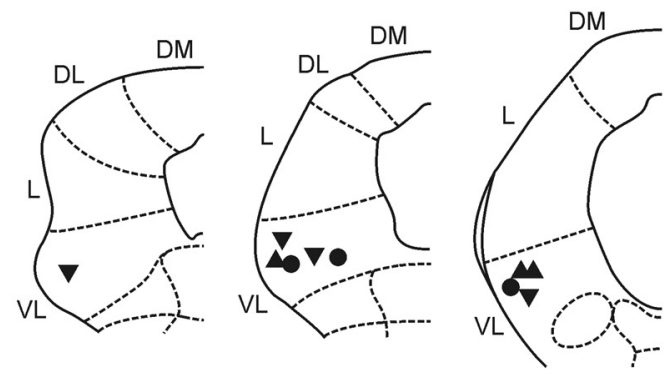

\section{$\Delta$ Acetone}

$\nabla$ Ethyl chloride

- Acetone + ethyl chloride

$-7.56$
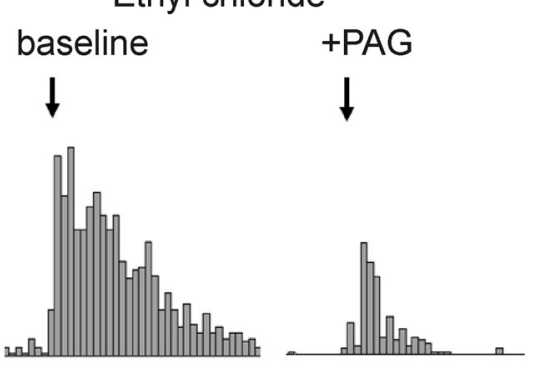

Figure 5. Noxious cold-evoked activity is selectively inhibited in wide-dynamic-range class 2 neurons by PAG stimulation. $A$, Typical examples of a class 2 neuron response to acetone and EC [recorded from the same neuron; peristimulus time histograms (spikes per $1 \mathrm{~s}$ bin) are shown] before and after PAG stimulation. $\boldsymbol{B}$, The effect of VL-PAG stimulation on class 2 neuronal responses to acetone $(n=6)$ and EC $(n=7)$. Data are expressed as mean \pm SEM of normalized spike counts in response to acetone or EC. Statistical analysis compared post-DLH (PAG) and recovery (PAG $+5 \mathrm{~min}, \mathrm{PAG}+10 \mathrm{~min}, \mathrm{PAG}+15 \mathrm{~min}$ ) groups with baseline responses using Kruskal-Wallis test followed by Dunn's multiple comparison test; $\mathrm{ns}, p>0.05 ; * * 0.01$. C, Injection sites in the PAG from which the effects of PAG stimulation on acetone- and/or EC-evoked responses were tested; coordinates are relative to bregma (Paxinos and Watson, 2005). DM, Dorsomedial; DL, dorsolateral; L, lateral; VL, ventrolateral.

(37 $\pm 8 \%, p<0.05$ compared with baseline, Kruskal-Wallis test, and Dunn's multiple comparison test) (Fig. 5). This suggests that different mechanisms may be responsible for descending modulation of class 3 neuronal responses (that remain depressed over the time period studied) compared with class 2 neuronal responses (that recover more rapidly). A total of 4/14 class 3 neurons (29\%) also responded to acetone (in addition to EC), and in one of these neurons (not positively identified as a projection neuron) the effects of descending control from the PAG were tested. VL-PAG stimulation abolished the response to acetone in this neuron (Fig. 6A,B). Noxious pinch-evoked activity was also tested in this neuron, which was similarly abolished by PAG stimulation (data not shown).

\section{Discussion}

An understanding of the spinal processing of cold sensory input, including its descending control, is essential not only in determining how forward transmission of cold signals may be altered in different behavioral states, but ultimately in the development of novel strategies for the treatment of aberrant cold sensations that accompany pathological pain states. Here, we show that spinal responses to cold can be powerfully modulated by descending control systems originating in the PAG and that this control selectively modulates transmission of noxious versus innocuous information.

\section{Neural mechanisms of cold somatosensation}

Two different intensities of cold stimuli were used to investigate central processing of both innocuous and noxious cold, providing novel information regarding mechanisms underlying behavioral responses to cold. Acetone produced only mild cooling of the skin and never evoked withdrawal reflexes in lightly anesthetized rats, consistent with observations that normal rats show little or no response to acetone, and with the use of acetone as a test for cold allodynia following neuropathy (Choi et al., 1994; Decosterd and Woolf, 2000; Kim et al., 2009; Hulse et al., 2010). Together with reports from human studies that temperatures between 15 and $20^{\circ} \mathrm{C}$ are perceived as cool (Greenspan et al., 1993), this suggests that acetone produces predominantly innocuous cooling. In contrast, EC produced a more intense cold stimulus, generating skin surface temperatures approaching $0^{\circ} \mathrm{C}$ in the present study, and its application consistently evoked withdrawal reflexes accompanied by increases in blood pressure. Together, this indicates that EC is a noxious cold stimulus. EC has been used previously in behavioral studies, and similar temperature changes have been reported (Hao et al., 1996, 1999; Sjolund et al., 1998). These temperature changes are sufficiently cold to activate populations of nociceptive afferents (LaMotte and Thalhammer, 1982; Lynn and Carpenter, 1982; Leem et al., 1993; Simone and Kajander, 1996, 1997). However, temperatures reported to produce cold pain sensation in humans and nociceptive behaviors in animals are highly variable, likely due to the wide variety of stimuli used and responses measured, making comparison between studies difficult. In humans, temperatures of $\sim 10-15^{\circ} \mathrm{C}$ and below are reported to evoke noxious sensations (Chery-Croze, 1983; Yarnitsky and Ochoa, 1990; Davis, 1998; Gottrup et al., 1998; Harrison and Davis, 1999). We observed cold-evoked withdrawal in lightly anesthetized rats at skin surface temperatures of $\sim 6^{\circ} \mathrm{C}$, which is consistent with 
Allchorne et al. (2005), who defined cold threshold in normal rats at $5^{\circ} \mathrm{C}$, and Jasmin et al. (1998), who reported nocifensive behaviors $<3^{\circ} \mathrm{C}$. Using operant assays, $\mathrm{Vi}-$ erck et al. (2004) reported thresholds for "lick/guard/jump" behaviors at $\sim 4^{\circ} \mathrm{C}$; however, escape behaviors were observed at much warmer temperatures $\left(\sim 16^{\circ} \mathrm{C}\right)$.

The present study provides novel information regarding the population of spinal neurons excited by acetone and EC, both of which are used in behavioral pain studies. We found that the majority of class 1 and 2 neurons are cold responsive, while half of class 3 neurons were not. Consistent with our data, Khasabov et al. (2001) found that an almost identical proportion of wide-dynamic-range (class 2) neurons responded to cold delivered via a Peltier thermode, which encoded intensity to noxious cold over a broad range of temperatures. Furthermore, functional anatomical studies have shown that hindpaw and facial cold stimulation evoke intensitydependent Fos expression in dorsal horn neurons, including NK-1-positive lamina I projection neurons, which are believed to form spinal-brainstem feedback loops with important roles in the development of chronic pain states (Strassman et al., 1993; Abbadie et al., 1994; Doyle and Hunt, 1999; Suzuki et al., 2002; Todd et al., 2005). However, in contrast to our findings, Khasabov et al. (2001) found a higher proportion of cold-responsive class 3 neurons, but around half $(42 \%)$ only responded to cooling $<0^{\circ} \mathrm{C}$. Many cold-sensitive nociceptors have thresholds of $<0^{\circ} \mathrm{C}$ (Simone and Kajander, 1996; 1997) (although this could be in response to tissue damage). It is possible, therefore, that the cold "insensitive" class 3 neurons described here are cold responsive but with thresholds at very low temperatures; therefore, the stimulus we used would not have produced cooling sufficient to activate them.

A seemingly unexpected finding was that some nociceptive-specific neurons (classified by standard mechanical methods) responded to acetone application, which is generally considered to be innocuous. However, this activity may be due to nociceptive input, since the temperatures produced by acetone application are sufficient to activate nociceptive afferents with cold thresholds at relatively warm temperatures $\left(>20^{\circ} \mathrm{C}\right.$ ) (Cain et al., 2001; Campero et al., 1996; Simone and Kajander, 1996). Any class 3 neurons receiving afferent input from these lowerthreshold nociceptors could therefore be acetone responsive. If this is the case, and given the small proportion of nociceptors with thresholds of $>20^{\circ} \mathrm{C}$, it is not surprising that a small proportion of class 3 neurons were found to be activated by acetone application. An additional possibility is that information transmitted by nociceptive-specific class 3 neurons is interpreted by

C
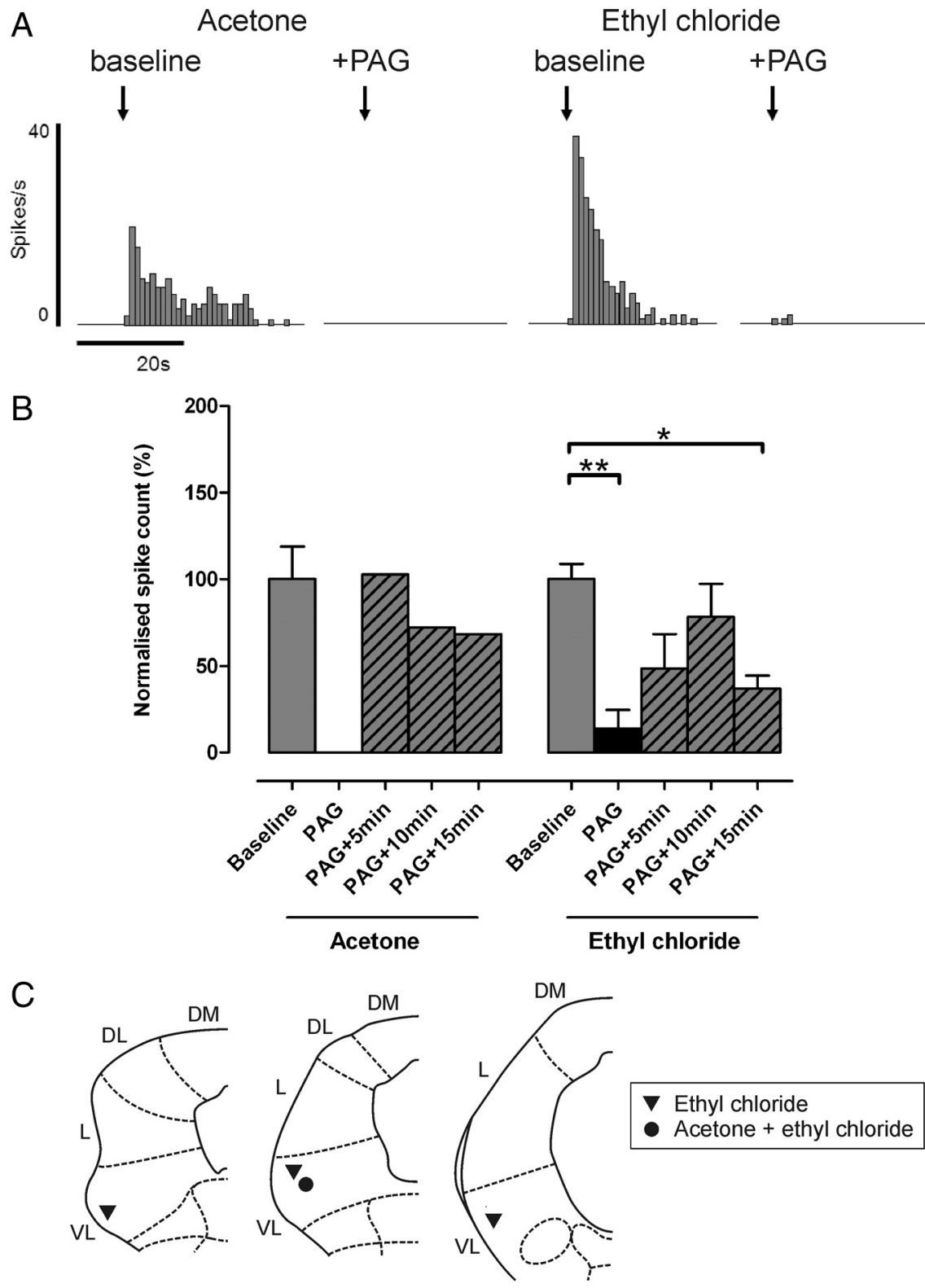

Ethyl chloride

- Acetone + ethyl chloride

Figure 6. Cold-evoked activity in class 3 nociceptive-specific neurons is inhibited by PAG stimulation. $A$, Examples of a class 3 neuron response to acetone and EC [recorded from the same neuron; peristimulus time histograms (spikes per $1 \mathrm{~s}$ bin) are shown] before and after PAG stimulation. $\boldsymbol{B}$, The effect of VL-PAG stimulation on mean class 3 neuronal responses to acetone $(n=1)$ and EC $(n=4)$. Data are expressed as mean \pm SEM of normalized spike counts in response to acetone or EC. Statistical analysis compared post-DLH (PAG) and recovery (PAG +5 min, PAG +10 min, PAG +15 min) groups with baseline responses using Kruskal-Wallis test followed by Dunn's multiple comparison test; ${ }^{*} p<0.05$; ${ }^{* *} p<0.01$. C, Injection sites in the PAG from which the effects of PAG stimulation on acetone- and/or EC-evoked responses were tested; coordinates are relative to bregma (Paxinos and Watson, 2005); DM, Dorsomedial; DL, dorsolateral; L, lateral; VL, ventrolateral.

higher centers as nociceptive, regardless of the intensity of the input.

\section{Descending modulation of spinal cold processing}

Stimulation of descending pathways from the PAG has been shown to powerfully modulate the responses of spinal neurons to peripheral mechanical and thermal (heat) stimulation in vivo (Mayer et al., 1971; Jones and Gebhart, 1988; Sandkuhler et al., 1991; Waters and Lumb, 1997, 2008; McMullan and Lumb, 2006b). This modulatory control is selective for responses to noxious versus innocuous stimulus intensities (Bennett and Mayer, 
1979; Gebhart et al., 1983; Sandkuhler et al., 1991; Waters and Lumb, 1997). We therefore hypothesized that this selective control might also extend to the modality of cold and tested this directly by examining whether acetone- and EC-evoked responses of spinal dorsal horn neurons were altered by PAG stimulation. We found that spinal processing of cold input can also be powerfully modulated by the PAG, and that this control differentiates between activity in nociceptive versus non-nociceptive circuits. This pattern of descending control therefore resembles that for other modalities and suggests that stimulus intensity (i.e., nociceptive versus non-nociceptive) rather than modality is the determining factor in whether spinal activity is subject to descending control.

Our findings do not, however, include cold-specific neurons that are not driven by mechanical stimulation (Han et al., 1998), since these would not have been encountered in our experiments. Given that these neurons will also contribute to cold-evoked behaviors, it will be important to investigate whether these cells are also modulated by descending pathways.

\section{Functional significance}

Temperature perception is vital for survival, providing environmental information that drives appropriate behavioral responses and, if necessary, escape from potentially harmful conditions. Knowledge of the central processing and modulation of cold sensory input is therefore important from a behavioral perspective, yet has received little attention. The present study demonstrates that descending control originating in the PAG can dramatically alter spinal responses to noxious cold input, leaving information regarding innocuous cold unaltered. In normal animals, modulation of spinal nociception by the VL-PAG is hypothesized to act as part of a coordinated passive coping strategy triggered by inescapable stressors (Keay and Bandler, 2001; Lumb, 2002; Lovick and Bandler, 2005). Selective inhibition of noxious information would allow an animal to respond appropriately to threatening or stressful situations without the distraction of nociceptive input, therefore enabling the generation of adaptive behaviors that are beneficial to survival.

Recent evidence suggests that the PAG retains the capacity to modulate cold responses in pathophysiological states, since electrical stimulation of the PAG attenuates enhanced behavioral responses to cold in neuropathic rats (Lee et al., 2000). It is now recognized that alterations in descending controls from brainstem centers, including the PAG, contribute to central sensitization and chronic pain states (Pertovaara et al., 1996; Urban and Gebhart, 1999; Pertovaara, 2000; Monhemius et al., 2001; Pertovaara and Wei, 2003; Vanegas and Schaible, 2004). Indeed, cold allodynia is dependent on descending control systems, since lidocaine block of the rostroventromedial medulla (RVM), a major relay of outflow from the PAG, attenuates cold allodynia in models of neuropathic injury (Taylor et al., 2007). Therefore, knowledge of descending influences on spinal processing of cold information is clinically important, given the heightened cold sensitivity frequently reported by patients with neuropathic injury (Ochoa and Yarnitsky, 1994; Jorum et al., 2003). We hypothesize that dysfunction of descending control from the PAG (decreased descending inhibition and/or increased facilitation), likely acting through the RVM and other medullary structures, contributes to cold allodynia, driving the exaggerated behavioral responses (Choi et al., 1994) and increased spinal neuronal excitability to cold (Brignell et al., 2008) observed in neuropathic pain models, in addition to peripheral mechanisms. Future studies investigating dynamic modulation of cold responsiveness in the transition from acute to chronic pain will provide important information regarding underlying neural mechanisms responsible for altered cold responses in pathological pain states.

\section{References}

Abbadie C, Honore P, Besson JM (1994) Intense cold noxious stimulation of the rat hindpaw induces c-fos expression in lumbar spinal cord neurons. Neuroscience 59:457-468.

Allchorne AJ, Broom DC, Woolf CJ (2005) Detection of cold pain, cold allodynia and cold hyperalgesia in freely behaving rats. Mol Pain 1:36.

Bandler R, Keay KA, Floyd N, Price J (2000) Central circuits mediating patterned autonomic activity during active vs passive emotional coping. Brain Res Bull 53:95-104.

Bennett GJ, Mayer DJ (1979) Inhibition of spinal cord interneurons by narcotic microinjection and focal electrical stimulation in the periaqueductal central gray matter. Brain Res 172:243-257.

Bessou P, Perl ER (1969) Response of cutaneous sensory units with unmyelinated fibers to noxious stimuli. J Neurophysiol 32:1025-1043.

Brignell JL, Chapman V, Kendall DA (2008) Comparison of icilin- and cold-evoked responses of spinal neurones, and their modulation of mechanical activity, in a model of neuropathic pain. Brain Res 1215:87-96.

Cain DM, Khasabov SG, Simone DA (2001) Response properties of mechanoreceptors and nociceptors in mouse glabrous skin: an in vivo study. J Neurophysiol 85:1561-1574.

Campero M, Serra J, Ochoa JL (1996) C-polymodal nociceptors activated by noxious low temperature in human skin. J Physiol 497:565-572.

Carlson JD, Maire JJ, Martenson ME, Heinricher MM (2007) Sensitization of pain-modulating neurons in the rostral ventromedial medulla after peripheral nerve injury. J Neurosci 27:13222-13231

Carrive P (1993) The periaqueductal gray and defensive behavior: functional representation and neuronal organization. Behav Brain Res 58:27-47.

Carstens E, Hartung M, Stelzer B, Zimmermann M (1990) Suppression of a hind limb flexion withdrawal reflex by microinjection of glutamate or morphine into the periaqueductal gray in the rat. Pain 43:105-112.

Chery-Croze S (1983) Relationship between noxious cold stimuli and the magnitude of pain sensation in man. Pain 15:265-269.

Choi Y, Yoon YW, Na HS, Kim SH, Chung JM (1994) Behavioral signs of ongoing pain and cold allodynia in a rat model of neuropathic pain. Pain 59:369-376.

Christensen BN, Perl ER (1970) Spinal neurons specifically excited by noxious or thermal stimuli: marginal zone of the dorsal horn. J Neurophysiol 33:293-307.

Colburn RW, Lubin ML, Stone DJ Jr, Wang Y, Lawrence D, D’Andrea MR, Brandt MR, Liu Y, Flores CM, Qin N (2007) Attenuated cold sensitivity in TRPM8 null mice. Neuron 54:379-386.

Davis KD (1998) Cold-induced pain and prickle in the glabrous and hairy skin. Pain 75:47-57.

Decosterd I, Woolf CJ (2000) Spared nerve injury: an animal model of persistent peripheral neuropathic pain. Pain 87:149-158.

Doyle CA, Hunt SP (1999) A role for spinal lamina I neurokinin-1-positive neurons in cold thermoreception in the rat. Neuroscience 91:723-732.

Fardin V, Oliveras JL, Besson JM (1984) A reinvestigation of the analgesic effects induced by stimulation of the periaqueductal gray matter in the rat. I. The production of behavioral side effects together with analgesia. Brain Res 306:105-123.

Gebhart GF, Sandkuhler J, Thalhammer JG, Zimmermann M (1983) Quantitative comparison of inhibition in spinal cord of nociceptive information by stimulation in periaqueductal gray or nucleus raphe magnus of the cat. J Neurophysiol 50:1433-1445.

Gottrup H, Nielsen J, Arendt-Nielsen L, Jensen TS (1998) The relationship between sensory thresholds and mechanical hyperalgesia in nerve injury. Pain 75:321-329.

Greenspan JD, Taylor DJ, McGillis SL (1993) Body site variation of cool perception thresholds, with observations on paradoxical heat. Somatosens Mot Res 10:467-474.

Han ZS, Zhang ET, Craig AD (1998) Nociceptive and thermoreceptive lamina I neurons are anatomically distinct. Nat Neurosci 1:218-225.

Hao JX, Yu W, Xu XJ, Wiesenfeld-Hallin Z (1996) Capsaicin-sensitive afferents mediate chronic cold, but not mechanical, allodynia-like behavior in spinally injured rats. Brain Res 722:177-180.

Hao JX, Xu IS, Xu XJ, Wiesenfeld-Hallin Z (1999) Effects of intrathecal 
morphine, clonidine and baclofen on allodynia after partial sciatic nerve injury in the rat. Acta Anaesthesiol Scand 43:1027-1034.

Harrison JL, Davis KD (1999) Cold-evoked pain varies with skin type and cooling rate: a psychophysical study in humans. Pain 83:123-135.

Heinricher MM, Tavares I, Leith JL, Lumb BM (2009) Descending control of nociception: specificity, recruitment and plasticity. Brain Res Rev 60:214-225.

Hulse R, Wynick D, Donaldson LF (2010) Intact cutaneous C fibre afferent properties in mechanical and cold neuropathic allodynia. Eur J Pain. Advance online publication. Retrieved February 24, 2010. doi:10.1016/ j.ejpain.2009.10.001.

Jasmin L, Kohan L, Franssen M, Janni G, Goff JR (1998) The cold plate as a test of nociceptive behaviors: description and application to the study of chronic neuropathic and inflammatory pain models. Pain 75:367-382.

Jones SL, Gebhart GF (1988) Inhibition of spinal nociceptive transmission from the midbrain, pons and medulla in the rat: activation of descending inhibition by morphine, glutamate and electrical stimulation. Brain Res 460:281-296.

Jorum E, Warncke T, Stubhaug A (2003) Cold allodynia and hyperalgesia in neuropathic pain: the effect of $N$-methyl-D-aspartate (NMDA) receptor antagonist ketamine-a double-blind, cross-over comparison with alfentanil and placebo. Pain 101:229-235.

Keay KA, Bandler R (2001) Parallel circuits mediating distinct emotional coping reactions to different types of stress. Neurosci Biobehav Rev 25:669-678.

Khasabov SG, Cain DM, Thong D, Mantyh PW, Simone DA (2001) Enhanced responses of spinal dorsal horn neurons to heat and cold stimuli following mild freeze injury to the skin. J Neurophysiol 86:986-996.

Kim HY, Gwak YS, Shim I (2009) An electrophysiological method for quantifying neuropathic pain behaviors in rats: measurement of hindlimb withdrawal EMG magnitude. J Physiol Sci 59:473-476.

Koutsikou S, Parry DM, MacMillan FM, Lumb BM (2007) Laminar organization of spinal dorsal horn neurones activated by C- vs A-heat nociceptors and their descending control from the periaqueductal grey in the rat. Eur J Neurosci 26:943-952.

LaMotte RH, Thalhammer JG (1982) Response properties of high-threshold cutaneous cold receptors in the primate. Brain Res 244:279-287.

Lee BH, Park SH, Won R, Park YG, Sohn JH (2000) Antiallodynic effects produced by stimulation of the periaqueductal gray matter in a rat model of neuropathic pain. Neurosci Lett 291:29-32.

Leem JW, Willis WD, Chung JM (1993) Cutaneous sensory receptors in the rat foot. J Neurophysiol 69:1684-1699.

Leith JL, Wilson AW, Donaldson LF, Lumb BM (2007) Cyclooxygenase-1derived prostaglandins in the periaqueductal gray differentially control C- versus A-fiber-evoked spinal nociception. J Neurosci 27:11296-11305.

Lipski J (1981) Antidromic activation of neurones as an analytic tool in the study of the central nervous system. J Neurosci Methods 4:1-32.

Lipski J, Bellingham MC, West MJ, Pilowsky P (1988) Limitations of the technique of pressure microinjection of excitatory amino acids for evoking responses from localized regions of the CNS. J Neurosci Methods 26:169-179.

Lovick TA, Bandler R (2005) The organisation of the midbrain periaqueductal grey and the integration of pain behaviours. In: The neurobiology of pain (Hunt SP, Koltzenburg M, eds), pp 267-287. Oxford: Oxford UP.

Lumb BM (2002) Inescapable and escapable pain is represented in distinct hypothalamic-midbrain circuits: specific roles for Adelta- and C-nociceptors. Exp Physiol 87:281-286.

Lynn B, Carpenter SE (1982) Primary afferent units from the hairy skin of the rat hind limb. Brain Res 238:29-43.

Mayer DJ, Wolfle TL, Akil H, Carder B, Liebeskind JC (1971) Analgesia from electrical stimulation in the brainstem of the rat. Science 174:13511354.

McMullan S, Lumb BM (2006a) Midbrain control of spinal nociception discriminates between responses evoked by myelinated and unmyelinated heat nociceptors in the rat. Pain 124:59-68.

McMullan S, Lumb BM (2006b) Spinal dorsal horn neuronal responses to myelinated versus unmyelinated heat nociceptors and their modulation by activation of the periaqueductal grey in the rat. J Physiol 576:547-556.
McMullan S, Simpson DA, Lumb BM (2004) A reliable method for the preferential activation of C- or A-fibre heat nociceptors. J Neurosci Methods 138:133-139.

Menetrey D, Giesler GJ Jr, Besson JM (1977) An analysis of response properties of spinal cord dorsal horn neurones to nonnoxious and noxious stimuli in the spinal rat. Exp Brain Res 27:15-33.

Menetrey D, Chaouch A, Besson JM (1979) Responses of spinal cord dorsal horn neurones to non-noxious and noxious cutaneous temperature changes in the spinal rat. Pain 6:265-282.

Millan MJ (2002) Descending control of pain. Prog Neurobiol 66:355-474.

Monhemius R, Green DL, Roberts MH, Azami J (2001) Periaqueductal grey mediated inhibition of responses to noxious stimulation is dynamically activated in a rat model of neuropathic pain. Neurosci Lett 298:70-74.

Ochoa JL, Yarnitsky D (1994) The triple cold syndrome: cold hyperalgesia, cold hypoaesthesia and cold skin in peripheral nerve disease. Brain 117:185-197.

Paxinos G, Watson C (2005) The rat brain in stereotaxic coordinates. Sydney: Academic.

Pertovaara A (2000) Plasticity in descending pain modulatory systems. Prog Brain Res 129:231-242.

Pertovaara A, Wei H (2003) A dissociative change in the efficacy of supraspinal versus spinal morphine in the neuropathic rat. Pain 101:237-250.

Pertovaara A, Wei H, Hamalainen MM (1996) Lidocaine in the rostroventromedial medulla and the periaqueductal gray attenuates allodynia in neuropathic rats. Neurosci Lett 218:127-130.

Sandkuhler J, Willmann E, Fu QG (1991) Characteristics of midbrain control of spinal nociceptive neurons and nonsomatosensory parameters in the pentobarbital-anesthetized rat. J Neurophysiol 65:33-48.

Simone DA, Kajander KC (1996) Excitation of rat cutaneous nociceptors by noxious cold. Neurosci Lett 213:53-56.

Simone DA, Kajander KC (1997) Responses of cutaneous A-fiber nociceptors to noxious cold. J Neurophysiol 77:2049-2060.

Sjolund KF, von Heijne M, Hao JX, Xu XJ, Sollevi A, Wiesenfeld-Hallin Z (1998) Intrathecal administration of the adenosine Al receptor agonist R-phenylisopropyl adenosine reduces presumed pain behaviour in a rat model of central pain. Neurosci Lett 243:89-92.

Strassman AM, Vos BP, Mineta Y, Naderi S, Borsook D, Burstein R (1993) Fos-like immunoreactivity in the superficial medullary dorsal horn induced by noxious and innocuous thermal stimulation of facial skin in the rat. J Neurophysiol 70:1811-1821.

Suzuki R, Morcuende S, Webber M, Hunt SP, Dickenson AH (2002) Superficial NK1-expressing neurons control spinal excitability through activation of descending pathways. Nat Neurosci 5:1319-1326.

Taylor BK, Abhyankar SS, Vo NT, Kriedt CL, Churi SB, Urban JH (2007) Neuropeptide $\mathrm{Y}$ acts at $\mathrm{Y} 1$ receptors in the rostral ventral medulla to inhibit neuropathic pain. Pain 131:83-95.

Todd AJ, Spike RC, Young S, Puskar Z (2005) Fos induction in lamina I projection neurons in response to noxious thermal stimuli. Neuroscience 131:209-217.

Urban MO, Gebhart GF (1999) Supraspinal contributions to hyperalgesia. Proc Natl Acad Sci U S A 96:7687-7692.

Vanegas H, Schaible HG (2004) Descending control of persistent pain: inhibitory or facilitatory? Brain Res Brain Res Rev 46:295-309.

Vierck CJ Jr, Kline Rt, Wiley RG (2004) Comparison of operant escape and innate reflex responses to nociceptive skin temperatures produced by heat and cold stimulation of rats. Behav Neurosci 118:627-635.

Waters AJ, Lumb BM (1997) Inhibitory effects evoked from both the lateral and ventrolateral periaqueductal grey are selective for the nociceptive responses of rat dorsal horn neurones. Brain Res 752:239-249.

Waters AJ, Lumb BM (2008) Descending control of spinal nociception from the periaqueductal grey distinguishes between neurons with and without C-fibre inputs. Pain 134:32-40.

Willis WD, Coggeshall RE (1991) Sensory mechanisms of the spinal cord, 2nd Ed. New York: Plenum.

Yarnitsky D, Ochoa JL (1990) Release of cold-induced burning pain by block of cold-specific afferent input. Brain 113:893-902. 